\title{
Integrated Skin Transcriptomics and Serum Multiplex Assays Reveal Novel Mechanisms of Wound Healing in Diabetic Foot Ulcers
}

\author{
Georgios Theocharidis, ${ }^{1}$ Dimitrios Baltzis, ${ }^{1}$ Matthieu Roustit, ${ }^{1}$ Ana Tellechea, ${ }^{1}$ Seema Dangwal, ${ }^{1}$ \\ Radhika S. Khetani, ${ }^{2}$ Bin Shu, ${ }^{1}$ Wanni Zhao, ${ }^{1}$ Jianfang Fu, ${ }^{1}$ Swati Bhasin, ${ }^{3}$ Antonios Kafanas, ${ }^{1}$ Daniel Hui, $, 45,6$ \\ Shannan Ho Sui, ${ }^{2}$ Nikolaos A. Patsopoulos, ${ }^{4,5,6}$ Manoj Bhasin, ${ }^{3}$ and Aristidis Veves ${ }^{1}$
}

Diabetes 2020;69:2157-2169 | https://doi.org/10.2337/db20-0188

\begin{abstract}
Nonhealing diabetic foot ulcers (DFUs) are characterized by low-grade chronic inflammation, both locally and systemically. We prospectively followed a group of patients who either healed or developed nonhealing chronic DFUs. Serum and forearm skin analysis, both at the protein expression and the transcriptomic level, indicated that increased expression of factors such as interferon- $\gamma$ (IFN- $\gamma$ ), vascular endothelial growth factor, and soluble vascular cell adhesion molecule-1 were associated with DFU healing. Furthermore, foot skin single-cell RNA sequencing analysis showed multiple fibroblast cell clusters and increased inflammation in the dorsal skin of patients with diabetes mellitus (DM) and DFU specimens compared with control subjects. In addition, in myeloid cell DM and DFU upstream regulator analysis, we observed inhibition of interleukin-13 and IFN- $\gamma$ and dysregulation of biological processes that included cell movement of monocytes, migration of dendritic cells, and chemotaxis of antigen-presenting cells pointing to an impaired migratory profile of immune cells in DM skin. The SLCO2A1 and CYP1A1 genes, which were upregulated at the forearm of nonhealers, were mainly expressed by the vascular endothelial cell cluster almost exclusively in DFU, indicating a potential important role in wound healing. These results from integrated protein and transcriptome analyses identified individual
\end{abstract}

genes and pathways that can potentially be targeted for enhancing DFU healing.

Diabetic foot ulcers (DFUs) are a major problem that results in $>70,000$ lower-extremity amputations annually in the U.S. alone (1). Low-grade chronic inflammation and inability to progress to the proliferative phase of wound healing are main characteristics of nonhealing DFUs (2). Previous studies from our unit have shown that patients with nonhealing DFUs have increased levels of serum biomarkers of inflammation and increased degranulation of skin mast cells $(3,4)$. However, the underlying mechanisms and signaling pathways bridging vascular dysfunction, inflammation, and peripheral neuropathy remain partly unknown.

Recent advances in single-cell transcriptomics have allowed the characterization of gene expression in specific cell types, and this is valuable in dynamic situations, such as wound healing, that require well-coordinated actions by numerous cell types, including monocyte/macrophages, fibroblasts, endothelial cells, and keratinocytes (5). Investigating the heterogeneity of these cells can offer a thorough understanding of impaired wound healing pathophysiology.

\footnotetext{
${ }^{1}$ Joslin-Beth Israel Deaconess Foot Center and The Rongxiang Xu, MD, Center for Regenerative Therapeutics, Beth Israel Deaconess Medical Center, Harvard Medical School, Boston, MA

2Bioinformatics Core, Harvard T.H. Chan School of Public Health, Boston, MA ${ }^{3}$ Department of Medicine, Division of Interdisciplinary Medicine and Biotechnology, and Genomics, Proteomics, Bioinformatics and Systems Biology Center, Beth Israel Deaconess Medical Center, Harvard Medical School, Boston, MA

${ }^{4}$ Systems Biology and Computer Science Program, Ann Romney Center for Neurological Diseases, Department of Neurology, Brigham and Women's Hospital, Harvard Medical School, Boston, MA

5Division of Genetics, Department of Medicine, Brigham and Women's Hospital, Harvard Medical School, Boston, MA
}

${ }^{6}$ Broad Institute of Harvard and Massachusetts Institute of Technology, Cambridge, MA

Corresponding author: Aristidis Veves, aveves@bidmc.harvard.edu

Received 24 February 2020 and accepted 29 July 2020

This article contains supplementary material online at https://doi.org/10.2337/ figshare.12733235.

G.T. and D.B. contributed equally to this work as co-first authors.

(C) 2020 by the American Diabetes Association. Readers may use this article as long as the work is properly cited, the use is educational and not for profit, and the work is not altered. More information is available at https://www.diabetesjournals .org/content/license. 
In the current study, we prospectively followed a large group of patients with DFUs and aimed to identify systemic and local factors associated with DFU healing. To this end, we first evaluated systemic changes to serum cytokines and growth factors. In addition, in an effort to map the cellular landscape of DFUs, we performed bulk and single-cell transcriptome analysis of forearm and foot skin specimens from subjects without diabetes mellitus (DM) and patients with DM with and without DFU and interrogated changes in cell types with a pivotal role in DFU healing.

\section{RESEARCH DESIGN AND METHODS}

\section{Subjects}

Our experimental approach is outlined in Fig. 1. We enrolled 23 healthy subjects without DM, 50 patients with DM without DFU, and 39 patients with DM with DFU, all aged 21-80 years. Patients with DFU were followed for 12 weeks and were subdivided in two groups: those who healed their DFU (healers) $(n=15)$ or failed to heal their DFU (nonhealers) $(n=24)$ (Fig. 1A). Subjects who were smokers or had a history of myocardial ischemia, angina, macroalbuminuria, or any other serious illness were excluded. Patients with DFU with peripheral arterial occlusive disease or any illness that affects wound healing were excluded. At enrollment, two healers had a second DFU that had healed by the end of the study, while three nonhealers had a second DFU that had not healed. None of the participants had an additional ulcer in the 12 weeks before the start of the study that had already healed upon enrollment. The participants' clinical characteristics are presented in Table 1. The protocol was approved by the Beth Israel Deaconess Medical Center institutional review board, and all subjects provided written informed consent.

\section{Methods}

\section{Baseline Visit}

All participants were recruited and followed at the JoslinBeth Israel Deaconess Foot Center. Patients with DFUs were also seen biweekly until the exit visit at 12 weeks. All tests performed during the baseline visit were repeated at exit. Serum cytokines and growth factors were measured using a Luminex MAGPIX apparatus and Millipore multiplex panels.

\section{Skin Biopsies}

Two 2-mm forearm skin biopsies were collected from 14 control subjects, 37 patients with DM without DFU,
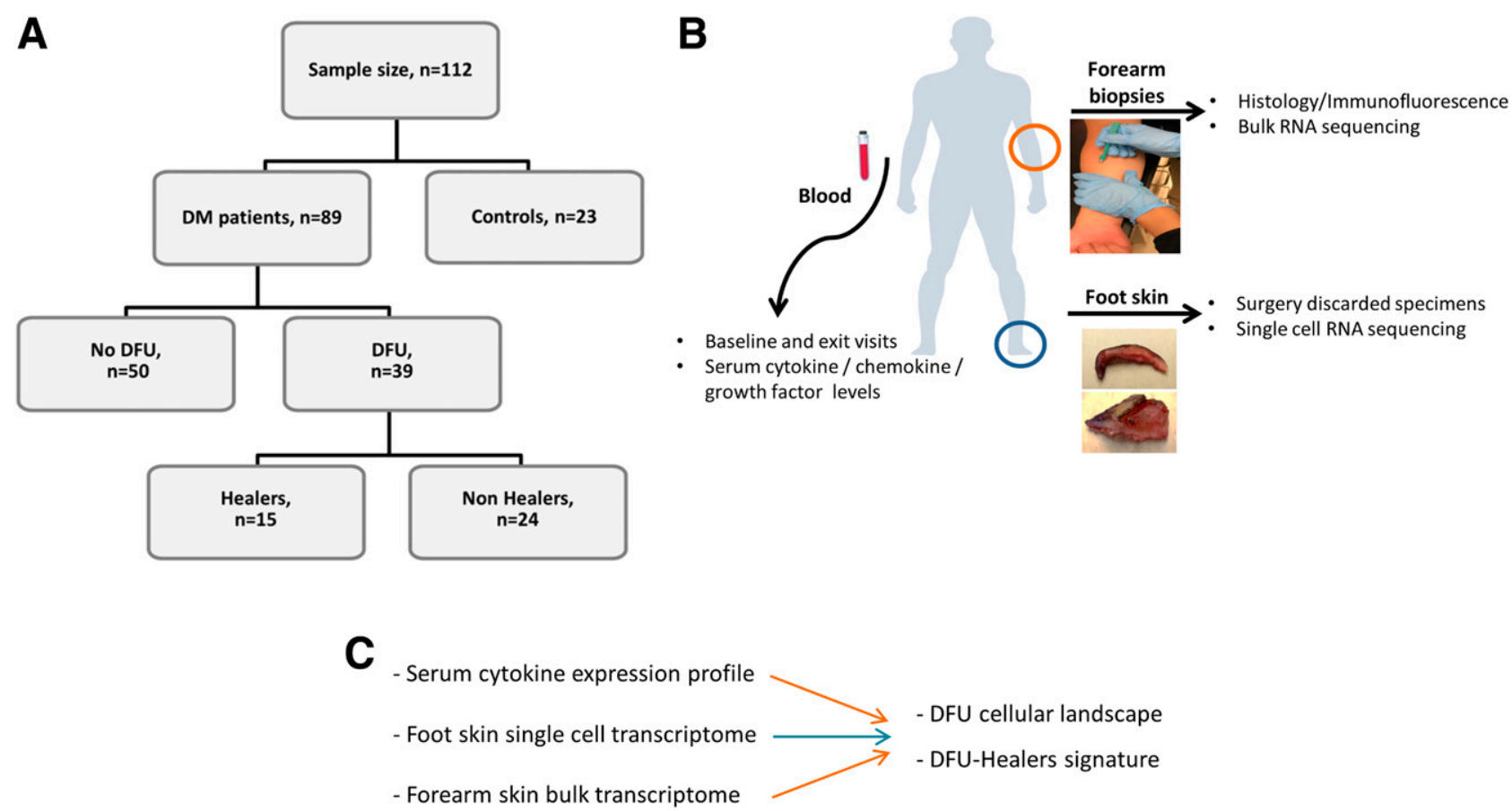

Figure 1-Study design, experimental approach, and analysis plan. A: Flowchart highlighting the prospective cohort studied: 112 total subjects, comprising 89 (79.5\%) patients with DM and 23 (20.5\%) control subjects without DM. Thirty-nine of the patients with DM had a DFU (43.8\%), 15 (38.5\%) of whom went on to heal their ulcer (healers). B: Schematic overview of the experimental procedures used in the study. Blood was collected at the baseline visit for all subjects and at the exit visit (either 12 weeks following baseline or 4 weeks posthealing for healers before completion of study) for patients with DFU. Serum cytokines, chemokines, and growth factors were quantitated with a Luminex apparatus or ELISA. Two 2-mm biopsies were obtained from the volar aspect of the forearm at the baseline visit and used for histology, immunofluorescence, and bulk RNA-seq experiments. Discarded skin specimens from foot surgeries were mechanically and enzymatically digested to create single-cell suspensions for capture with the 10× Genomics Chromium system for single-cell RNA-seq. C: Illustrative diagram of various analysis strategies. The results from multiplex arrays and transcriptome studies were used to generate the cellular landscape of foot skin and healers signature. 
Table 1-Clinical characteristics of the studied subjects

\begin{tabular}{|c|c|c|c|c|c|}
\hline & \multirow[b]{2}{*}{$\begin{array}{c}\text { Control subjects } \\
(n=23) \\
\end{array}$} & \multirow[b]{2}{*}{$\begin{array}{l}\text { Patients with DM } \\
(n=50)\end{array}$} & \multicolumn{2}{|c|}{ Subjects with DFU } & \multirow[b]{2}{*}{$P$ value } \\
\hline & & & $\begin{array}{l}\text { Healers } \\
(n=15)\end{array}$ & $\begin{array}{c}\text { Nonhealers } \\
(n=24)\end{array}$ & \\
\hline Age (years) & $57.3 \pm 10.6$ & $60.0 \pm 8.2$ & $50.1 \pm 9.0^{*} \dagger$ & $58.1 \pm 7.4 \ddagger$ & 0.003 \\
\hline Male sex & $20(87)$ & $31(62)$ & $12(80)$ & $18(75)$ & 0.14 \\
\hline Type 1 DM & - & $8(16)$ & $3(20)$ & $1(4.2)$ & 0.30 \\
\hline DM duration (years) & - & $15(8-30)$ & $15(5-38)$ & $20(5-25)$ & 0.98 \\
\hline Weight (kg) & $86.2(80-95)$ & $95.2(86-112)$ & $106.6(100-125)^{\star}$ & $97.1(87-109)$ & 0.049 \\
\hline BMI $\left(\mathrm{kg} / \mathrm{m}^{2}\right)$ & $27.9(26-29)$ & $30.8(27-37)$ & $32.4(30-37)^{\star}$ & $29.9(27-32)$ & 0.017 \\
\hline $\begin{array}{l}\text { Blood pressure }(\mathrm{mmHg}) \\
\text { Systolic } \\
\text { Diastolic }\end{array}$ & $\begin{array}{c}123(115-134) \\
77(70-85)\end{array}$ & $\begin{array}{c}120(117-130) \\
75(70-80)\end{array}$ & $\begin{array}{c}131.5(120-142) \\
72.5(70-80)\end{array}$ & $\begin{array}{c}130(120-146) \\
72.5(67-80)\end{array}$ & $\begin{array}{l}0.27 \\
0.68\end{array}$ \\
\hline Fasting glucose (mg/dL) & $93(84-103)$ & $128.5(96-195)^{\star}$ & $143(107-299)^{\star}$ & $134(94-188)$ & 0.006 \\
\hline $\mathrm{HbA}_{1 \mathrm{c}}(\%)$ & $5.6(5.5-5.9)$ & $7.7(6.6-8.4)^{\star}$ & $8.9(7.7-10.6)^{\star}$ & $8.4(6.8-9)^{\star}$ & $<0.001$ \\
\hline $\mathrm{HbA}_{1 \mathrm{c}}(\mathrm{mmol} / \mathrm{mol})$ & $38(37-41)$ & $61(49-68)^{*}$ & $74(61-92)^{\star}$ & $68(51-74)^{\star}$ & $<0.001$ \\
\hline Total cholesterol (mg/dL) & 201 (168-225) & $164.5(150-196)^{\star}$ & 169 (139-192) & $167(138-194)^{\star}$ & 0.006 \\
\hline LDL (mg/dL) & $104(83-135)$ & $84(66-99)^{\star}$ & $81(61-96)$ & $92(62-108)$ & 0.02 \\
\hline HDL (mg/dL) & $53(42-73)$ & $47(37-64)$ & $39(33-52)$ & $52(36-58)$ & 0.12 \\
\hline Triglycerides (mg/dL) & $124(95-190)$ & $121.5(84-202)$ & $135(101-300)$ & $138(108-222)$ & 0.50 \\
\hline ACE inhibitors & $3(13)$ & $21(42)$ & $7(46.7)$ & $9(37.5)$ & 0.24 \\
\hline AT- 1 blockers & $1(4.3)$ & $11(22)$ & $0(0)$ & $4(16.7)$ & 0.11 \\
\hline$\beta$-Blockers & $4(17.4)$ & $17(34)$ & $4(26.7)$ & $6(25)$ & 0.85 \\
\hline Ca antagonists & $2(8.7)$ & $13(26)$ & $4(26.7)$ & $4(16.7)$ & 0.6 \\
\hline Diuretics & $0(0)$ & $15(30)$ & $3(20)$ & $8(33.3)$ & 0.017 \\
\hline Statins & $2(8.7)$ & $30(60)^{\star}$ & $7(46.7)^{\star}$ & $16(66.7)^{\star}$ & $<0.001$ \\
\hline Insulin & & $26(52)$ & $11(73.3)$ & $10(41.7)$ & 0.3 \\
\hline Metformin & & $26(52)$ & $5(33.3)$ & $10(41.7)$ & 0.37 \\
\hline Sulfonylureas & & $8(16)$ & $2(13.3)$ & $8(33.3)$ & 0.12 \\
\hline DPP-4 inhibitors & & $2(4)$ & $0(0)$ & $0(0)$ & 1 \\
\hline GLP-1 analogs & & $4(8)$ & $0(0)$ & $1(4.2)$ & 0.82 \\
\hline ABI & - & $0.98 \pm 0.12$ & $1.04 \pm 0.14$ & $0.98 \pm 0.10$ & 0.26 \\
\hline NSS & - & $4(1-8)$ & $8(7-11) \dagger$ & $3(1-7) \ddagger$ & 0.002 \\
\hline NDS & - & $8.79 \pm 6.72$ & $15.89 \pm 7.09 \dagger$ & $13.41 \pm 7.03 \dagger$ & 0.002 \\
\hline VPT & - & $28 \pm 15$ & $37 \pm 18$ & $36 \pm 15$ & 0.052 \\
\hline SW & - & $4.93(4.74-6.45)$ & $6.45(5.46-7) \dagger$ & $6.27(5.46-7) \dagger$ & 0.001 \\
\hline
\end{tabular}

Data are mean $\pm \mathrm{SD}$, median (interquartile range), or $n(\%)$. ABI, ankle-brachial pressure index; AT-1, angiotensin II receptor-1; DPP-4, dipeptidyl peptidase 4; GLP-1, glucagon-like peptide 1; NDS, neuropathy disability score; NSS, neuropathy symptom score; SW, Semmes-Weinstein monofilament; VPT, vibration perception threshold. *Versus control subjects without DM. ${ }^{\top}$ Versus DM. ${ }^{\ddagger} P<0.05$ vs. healers.

13 healers, and 18 nonhealers. Mast cell and macrophage evaluation was performed as previously described (4). The antibodies used are listed in Supplementary Table 1.

\section{Bulk RNA Sequencing}

RNA was isolated from snap frozen forearm punch biopsies using the miRNeasy Kit (QIAGEN). Libraries were generated with NEBNext Ultra II RNA Library Prep Kit (New England Biolabs) per manufacturer's instructions and sequenced on an Illumina HiSeq 2000 system with a $2 \times 150$ paired-end run. Analyses were performed following best practices (6).

\section{Generation of Single-Cell Skin Digests}

Discarded surgical foot skin specimens were kept in sterile PBS on ice and quickly processed. Tissue was washed in ethanol, minced, and then placed in an enzyme cocktail of $4 \mathrm{mg} / \mathrm{mL}$ Collagenase P (Roche), $4 \mathrm{mg} / \mathrm{mL}$ Dispase II (Thermo Fisher Scientific), and $1 \mathrm{mg} / \mathrm{mL}$ DNase I (STEMCELL Technologies) in DMEM (Gibco) on a shaker for $2 \mathrm{~h}$ at $37^{\circ} \mathrm{C}$. Cell suspensions were filtered through 70 - and $40-\mu \mathrm{m}$ cell strainers and centrifuged. Cell pellets were resuspended in $0.04 \%$ UltraPure BSA (Thermo Fisher Scientific), and the concentration was adjusted to 1,000-2,000 cells $/ \mu \mathrm{L}$ for single-cell capture. 


\section{Single-Cell RNA Sequencing}

Cells were processed on a Chromium Controller with Chromium Single Cell 3' Reagent Kit (v2 Chemistry; $10 \times$ Genomics) per manufacturer's instructions. Libraries were generated following the $10 \times$ Genomics protocol and sequenced on Illumina NextSeq 500. Seurat 2.3.4 was used for analysis (7). Ingenuity Pathway Analysis (IPA) 8.0 software (Ingenuity Systems) was used for functional analyses (8).

\section{Statistical Analysis}

Statistical analyses were performed using SPSS version 23.0 software (IBM Corporation). Continuous data were expressed as mean $\pm \mathrm{SD}$ or as the median (interquartile range) when the distribution was not normal. Betweengroup comparisons were analyzed by one-way ANOVA. Two-way repeated-measures ANOVA was used to compare continuous variables over time between healers and nonhealers. ANCOVA was used for comparisons of continuous variables using age as a covariate. Exploratory principal component analysis (PCA) with direct oblimin rotation was conducted to identify combinations of variables that explain the greatest variance. Factors associated with time to healing were analyzed using a Cox proportional hazards model, with age as covariate. $P<0.05$ was considered significant.

\section{Data and Resource Availability}

Sequencing data are accessible through Gene Expression Omnibus series accession number GSE143735. Any additional data are available from the corresponding author upon reasonable request.

\section{RESULTS}

\section{Serum Cytokines, Chemokines, and Growth Factors}

The differences observed in serum cytokines, chemokines, biomarkers of endothelial function, and growth factors at baseline among the four groups are summarized in Supplementary Table 2. Of interest, soluble vascular cell adhesion molecule (sVCAM), vascular endothelial growth factor (VEGF), and interferon- $\gamma$ (IFN- $\gamma$ ) were increased in DFU healers, while tumor necrosis factor- $\alpha$ (TNF- $\alpha$ ) was reduced. Because we observed a difference in age between healers and nonhealers, we conducted post hoc comparisons adjusted on age. These confirmed our results, with significantly increased levels of sVCAM $(P=0.02)$, VEGF $(P=0.016)$, and IFN- $\gamma(P=0.04)$ in healers. On the other hand, TNF- $\alpha(P=0.03)$ was increased in nonhealers. These biomarkers had predominant weight in the exploratory PCA, in which four subsets of serum markers explained $72 \%$ of the variance (Supplementary Fig. $1 A-D$ ).

Among patients with DFUs, a higher level of substance $\mathrm{P}$ at baseline was associated with a shorter time to complete healing $(P=0.024$, Cox regression model, adjusted on age) (Fig. 2A). Similarly, higher levels of IFN- $\gamma(P=$ $0.006)$ and VEGF $(P=0.038)$ were associated with improved healing (Fig. $2 B$ and $C$ ). Measurements at the exit visit showed a significant increase in concentrations of interleukin-8 (IL-8) $(P=0.017)$ and TNF- $\alpha(P=0.013)$ in healers (Fig. $2 D$ and $E$ ). Additionally, a trend toward decreased soluble intracellular adhesion molecule 1 (sICAM-1) and sVCAM- 1 was observed between the baseline and exit visits (Fig. $2 F$ and $G$ ).

No differences were observed in the micro- and macrovascular reactivity between healers and nonhealers (Supplementary Table 3 ). These results are compatible with previous studies in our unit that showed no association between vascular reactivity and DFU healing (3).

\section{Mast Cell Degranulation and Inflammatory Cells}

Because previous studies in our unit have reported that systemic changes are reflected at the skin of patients with DM, we first evaluated immune cells in forearm skin biopsies (4) (Supplementary Table 4). All DM groups showed an increased proportion of degranulated mast cells, and they tended to be lower in healers (Supplementary Fig. 2A). Small round cells counted from hematoxylineosin-stained specimens were confirmed to be inflammatory as positive for leukocyte marker $\mathrm{CD} 45 \mathrm{RO}$ and dendritic cell marker factor XIIIa (3). Using the same techniques, the number of inflammatory cells was increased in patients with DM and healers compared with control subjects, while they were not in the nonhealers. In addition, we found increased numbers of M2-associated macrophages in healers compared with nonhealers (Supplementary Fig. $2 B-D$ ).

\section{Bulk RNA Sequencing Reveals Transcriptome Signature of Healers}

To establish whether DFU healing outcome was reflected at a nonulcerative skin site, we used bulk RNA sequencing (RNA-seq) to profile mRNA expression in forearm biopsies of healers $(n=5)$, nonhealers $(n=4)$, or patients with DM without DFU $(n=4)$. Patient details are listed in Supplementary Table 5. According to transcriptome data analysis with a false discovery rate $<0.05$ and log-twofold change $>0.5$, a total of 25 genes ( 3 upregulated) were differentially expressed when comparing nonhealers and healers, 916 (530 upregulated) in healers compared with patients with DM, and 160 (89 upregulated) in nonhealers compared with patients with DM (Fig. $3 A$ ). The differentially expressed genes are listed in Supplementary Tables 6-8. Noteworthy genes that increased in healers were inflammation-associated molecules CCL19 and $\beta$-defensin 124 (DEFB124) as well as extracellular matrix (ECM)-linked proteins tenascin X (TNXB) and MMP2. For nonhealers, upregulated genes were cytochrome P450 family member (CYP1A1) and prostaglandin transporter (SLCO2A1) (Fig. 3B). Moreover, IPA most significantly implicated pathways in nonhealers versus healers were acute phase response signaling and agranulocyte/granulocyte adhesion and diapedesis (Fig. 3C). IPA for prediction of upstream regulators revealed IFNG and TGFB1 as central players in nonhealers versus healers (Fig. 3D). It also showed 

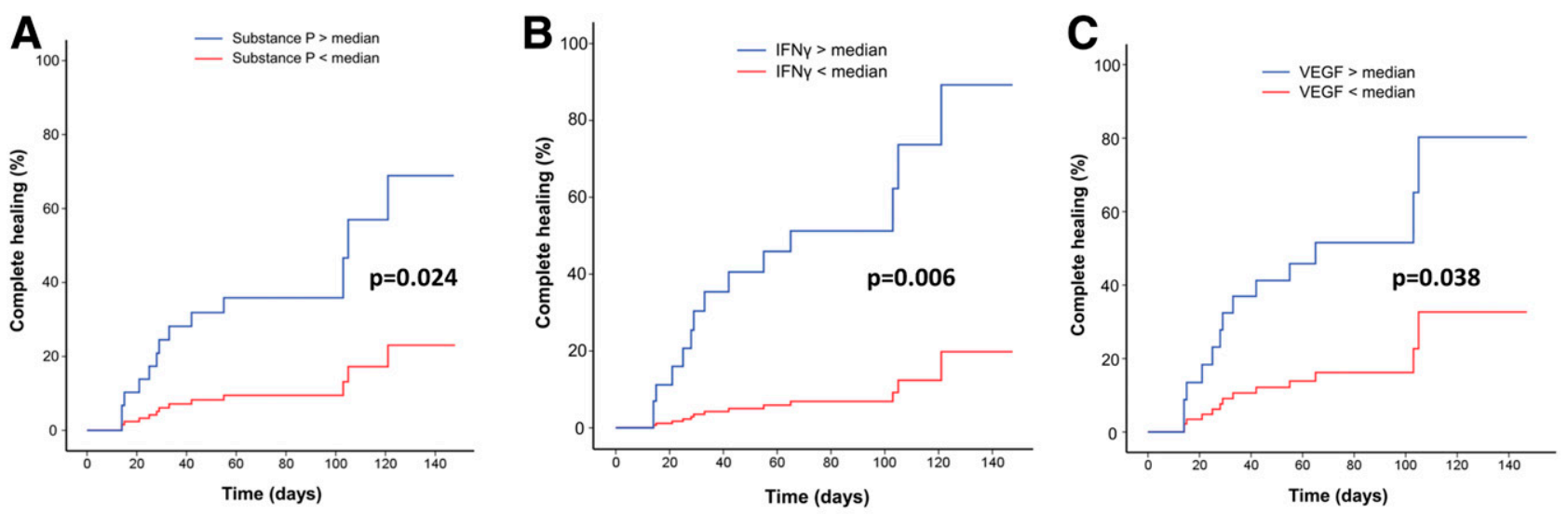

D
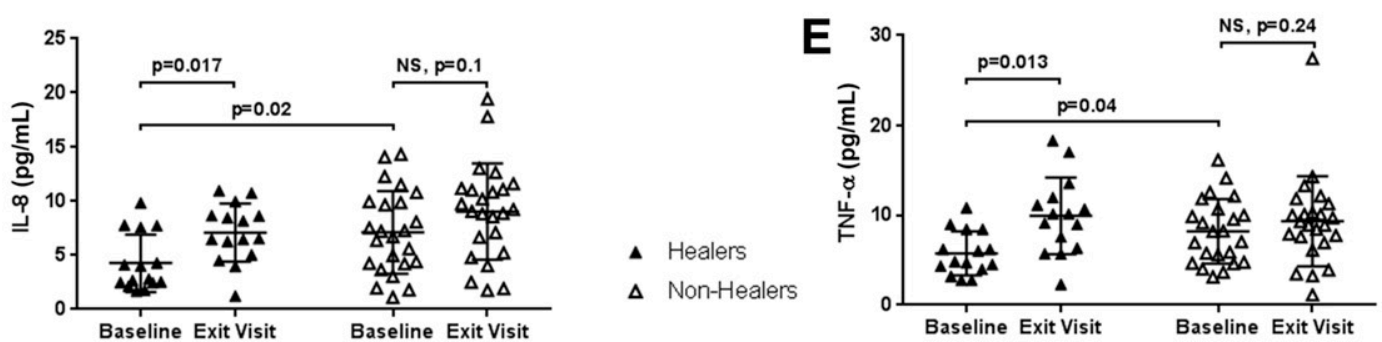

$\mathbf{F}$
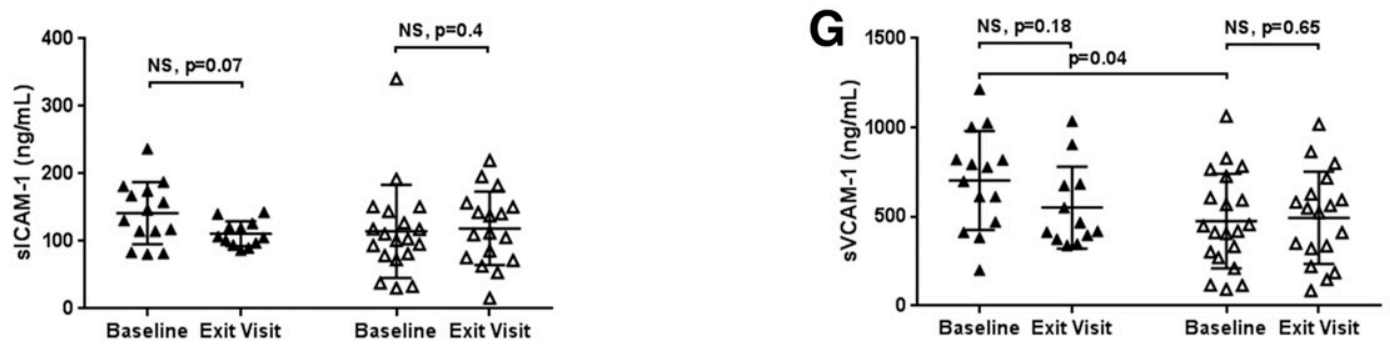

Figure 2-Serum profiles of selected inflammatory cytokines, chemokines, and biomarkers of endothelial function for healers and nonhealers at baseline and exit. $A-C$ : Time to complete healing according to baseline serum levels for substance $\mathrm{P}(A)$, IFN- $\gamma(B)$, and VEGF $(C)$ quantitated with ELISA or Luminex assay and applied to a Cox regression model adjusted on age with $P$ values shown. $D-G$ : Baseline and exit levels between healers and nonhealers for IL-8 $(D)$, TNF- $\alpha(E)$, sICAM-1 $(F)$, and sVCAM-1 $(G)$, measured with multiplex cytokine kits on the Luminex platform. Data are mean \pm SD ( $n=14-24$ patients); comparisons and significances are displayed and calculated with two-way repeated-measures ANOVA with Fisher post hoc test. NS, not significant.

activation of cell cycle regulator TP53 and mitochondrial metabolism mediator DAP3 in healers versus patients with DM (Fig. 3E) and activation of IL13 as well as implication of IL15, IL4R, and VEGFA in nonhealers versus patients with DM (Fig. 3F). IPA network analysis for nonhealers versus healers highlighted a gene interaction network with MMP2 and $\beta$-catenin as key nodes, while top-associated diseases and functions were cellular development, growth and proliferation, and movement (Supplementary Fig. 3).

\section{Single-Cell Transcriptomics of Foot Skin}

We next focused at the foot level, where DFUs develop, and performed single-cell RNA-seq. To this end, we studied foot dorsum skin specimens from elective surgeries of four subjects without DM and four patients with DM without DFU. We also studied discarded specimens from four patients with DFU who had their ulcers and adjacent nonulcerated tissue surgically excised. Cells were isolated without prepurification or enrichment, after enzymatic digestion. Patient demographics and surgery details are listed in Supplementary Table 9.

The non-DM samples comprised 3,865 cells in a total of 10 clusters as illustrated in Fig. $4 A$, $t$-distributed stochastic neighbor embedding ( $t$-SNE), and uniform manifold approximation and projection (UMAP) (Supplementary Fig. $4 C$ ) visualizations. Looking at the most expressed genes per cluster, we categorized them according to established markers. All the markers per cluster are shown in Supplementary Table 10. The most abundant cell types were fibroblasts expressing markers collagen type I $\alpha-1$ (COL1A1) and fibronectin (FN1) (Fig. 4D) in five different clusters that appeared close together in $t$-SNE and UMAP (Fibro1-5). Cluster Fibro1 was enriched for adipocyte markers, including APOE (9) and CFD (10). Fibro2 was characterized by the presence of reticular 

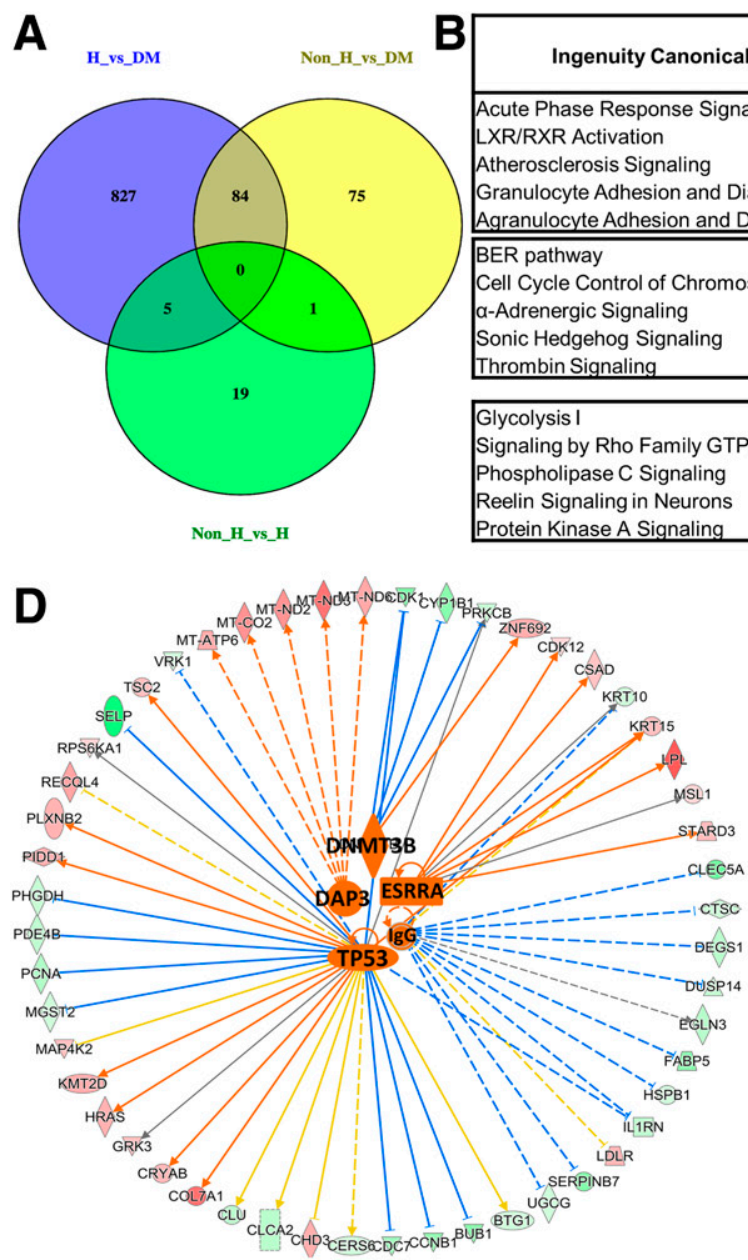

\begin{tabular}{|c|c|}
\hline Ingenuity Canonical Pathways & $-\log (p$-value $)$ \\
\hline $\begin{array}{l}\text { Acute Phase Response Signaling } \\
\text { LXR/RXR Activation } \\
\text { Atherosclerosis Signaling } \\
\text { Granulocyte Adhesion and Diapedesis } \\
\text { Agranulocyte Adhesion and Diapedesis } \\
\end{array}$ & $\begin{array}{l}3.23 \\
2.24 \\
2.24 \\
1.98 \\
1.93 \\
\end{array}$ \\
\hline $\begin{array}{l}\text { BER pathway } \\
\text { Cell Cycle Control of Chromosomal Replication } \\
\text { a-Adrenergic Signaling } \\
\text { Sonic Hedgehog Signaling } \\
\text { Thrombin Signaling }\end{array}$ & $\begin{array}{l}4.06 \\
3.24 \\
3.22 \\
2.91 \\
2.52 \\
\end{array}$ \\
\hline $\begin{array}{l}\text { Glycolysis I } \\
\text { Signaling by Rho Family GTPases } \\
\text { Phospholipase C Signaling } \\
\text { Reelin Signaling in Neurons } \\
\text { Protein Kinase A Signaling } \\
\end{array}$ & $\begin{array}{l}3.14 \\
2.66 \\
2.61 \\
2.54 \\
2.1\end{array}$ \\
\hline
\end{tabular}

E

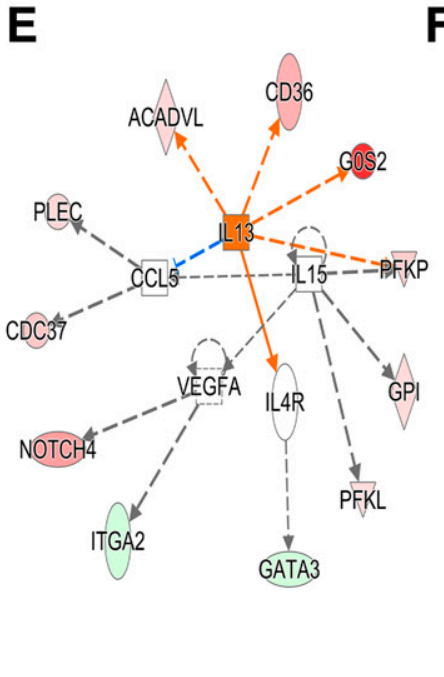

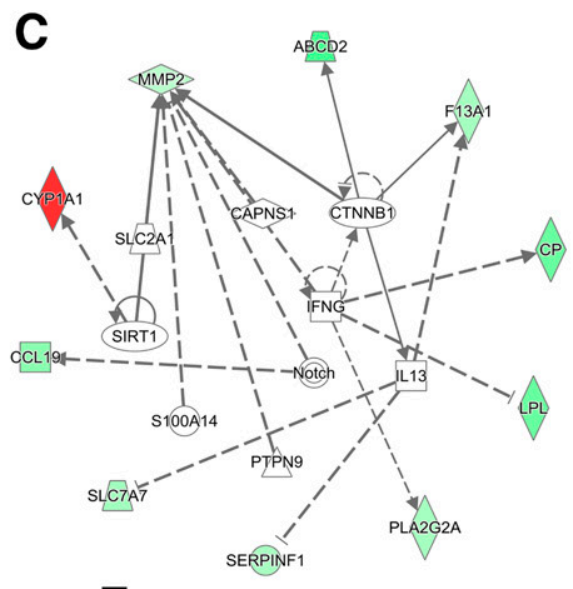

F Network Shapes

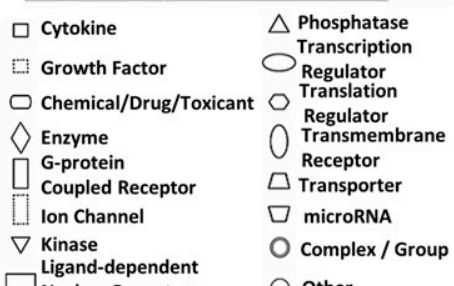

Figure 3-Bulk transcriptomic analysis of the forearm skin of patients with DM. $A$ : Venn diagram of significantly differentially expressed genes (DEGs) among the different comparisons of healers $(H)$ vs. DM, nonhealers (Non_H) vs. DM, and Non_H vs. H computed with a false discovery rate $<0.05$ and log-twofold change $>0.5$. Patients who healed $(n=5)$ or did not heal $(n=4)$ their ulcers and patients with DM without ulcers $(n=4)$ were analyzed. The sum of the numbers for each circle corresponds to the number of DEGs, while the overlapping numbers represent the mutual DEGs between different comparisons. $B$ : Top five enriched IPA canonical pathways ranked according to $P$ value (one-tailed Fisher exact test) for Non_H vs. $\mathrm{H}$ (top), $\mathrm{H}$ vs. DM (middle), and Non_H vs. DM (bottom) comparisons. C-E: IPA for prediction of upstream transcriptional regulators in Non_H vs. $\mathrm{H}(C), \mathrm{H}$ vs. DM $(D)$, and Non_H vs. DM $(E)$ comparisons reveals IFNG, Notch, and IL13 (C); DAP3, TP53, ESRRA, and DNMT3B (D); and IL13, IL15, IL4R, and VEGFA (E) as the top implicated regulators. F: Detailed legend explaining relationships and activation/inhibition in IPA upstream regulator results. BER, base excision repair; GTPase, guanosine triphosphatase; LXR/ $\mathrm{RXR}$, liver $\mathrm{X}$ receptor/retinoid X receptor.

fibroblast markers MFAP5 (11) and ELN (12), while Fibro3 contained a number of inflammatory markers, including CXC-type chemokines and IL6. In addition, Fibro4 exhibited enrichment of papillary fibroblast markers APCDD1 (13), COL6A1 (14), and COL23A1 (11). Finally, Fibro5 showed increased levels of Schwann cell marker CDH19 (15). Additional cell types were smooth muscle cells (SMCs) with markers $\alpha$-smooth muscle actin (ACTA2) (16) and transgelin (TAGLN) (17), vascular endothelial cells (VECs) (marker E-selectin [SELE] [18]), lymphatic endothelial cells (LECs) (marker lymphatic vessel endothelial hyaluronan receptor 1 [LYVE1] [19]), T cells (markers CD3 $\delta$-chain $[C D 3 D]$ and protein tyrosine phosphatase receptor type $C$ [PTPRC] [20]), and myeloid origin cells monocytes and macrophages (Mono/Macro) (markers
IL1B and lysozyme [LYZ] [21]). The heat map in Fig. $4 G$ depicts the top 10 genes per cluster and reveals efficient clustering. We note that no cells of epidermal origin were identified, most probably because of the skin processing, which did not include prior dermal-epidermal separation. However, the captured cells represent the major cell types present in the human dermis.

The DM samples consisted of 3,691 cells in 13 clusters (Fig. $4 B$ and E). All markers are shown in Supplementary Table 11, and Supplementary Fig. 5A depicts the top 10 genes per cluster. Fibroblasts again comprised five clusters (Fibro1-5), making them the most abundant cell type. Fibro1 was enriched for adipocyte markers, Fibro2 for papillary markers, and Fibro3 for reticular fibroblast markers, while Fibro4 featured high expression of a different set of 

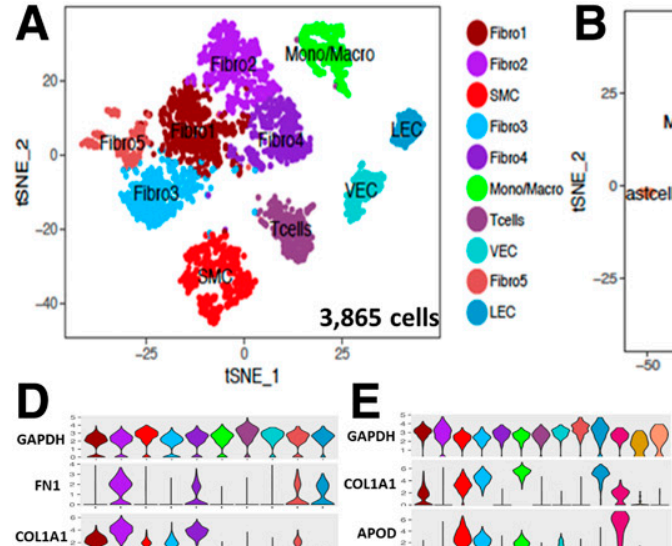

COL1A1 $\$+1+1+1+$
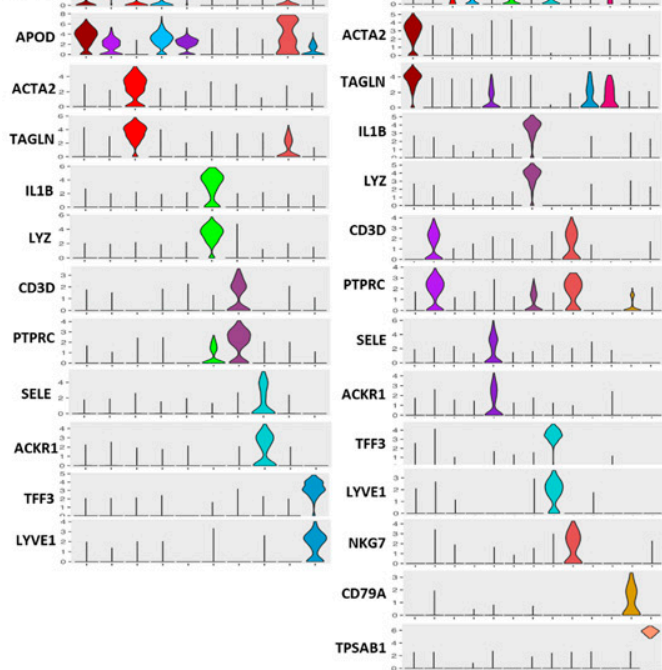

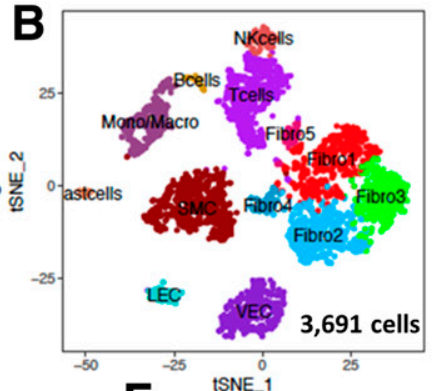

$\mathbf{F}$

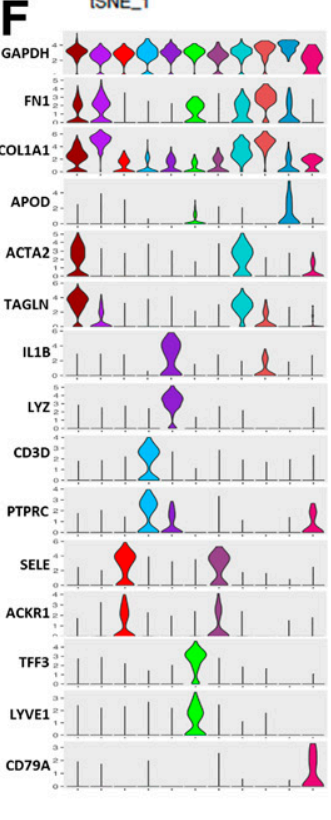

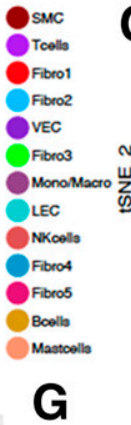

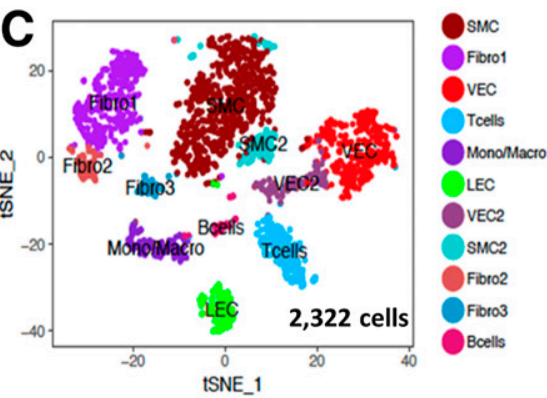

G

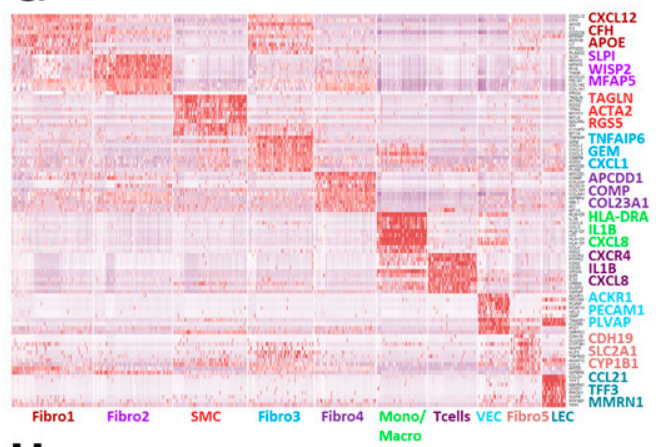

H

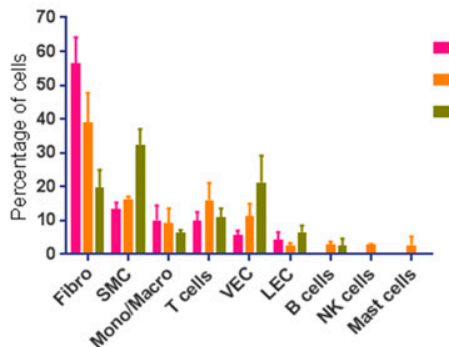

Figure 4-Single-cell RNA-seq analysis of lower-extremity skin samples reveals the cellular landscape of DM wound healing. A-C: Twodimensional plots derived in Seurat from nonlinear dimensional reduction by $t$-SNE technique of the single-cell transcriptomes of 3,865 cells from foot skin of four subjects without DM $(A), 3,691$ cells from foot skin of four patients with DM without DFUs $(B)$, and 2,322 cells from active DFUs of four patients $(C)$. Each dot represents a single cell. Clusters were colored according to the unsupervised clustering in Seurat and identified and annotated by using canonical marker genes for cell types enriched in each cluster. $D-F$ : Violin plots of representative marker genes showing their normalized expression ( $y$-axis) across all identified clusters $(x$-axis) per condition for subjects without $D M(D)$, patients with DM $(E)$, and subjects with DFU $(F)$. The colors of the violin plots are the same as their respective clusters in panels $A-C$. GAPDH shown as a housekeeping gene. FN1, COL1A1, and apolipoprotein $\mathrm{D}(A P O D)$ selected as fibroblast markers; $A C T A 2$ and TAGLN as SMC markers; IL1B and $L Y Z$ as Mono/Macro markers; $C D 3 D$ and PTPRC as T-cell markers; SELE and atypical chemokine receptor 1 (ACKR1) as VEC markers; trefoil factor 3 (TFF3) and LYVE1 as LEC markers; NKG7 as NK-cell marker; and CD79A B-cell marker and TPSAB1 as mast cell markers. G: Heat map depicting the top 10 (with the top 3 highlighted) differentially expressed genes in each cluster for subjects without DM. Each column represents a single cell, and each row represents an individual gene. Red corresponds to maximum relative gene expression. $H$ : Composition of identified cell types per condition. Data are mean $\pm \operatorname{SD}(n=4)$.

papillary markers, including WNT5A and type VII collagen (COL7A1). Fibro5 contained Schwann cell markers such as FOXS1 (22). Similar to patients without DM, other identified clusters were SMCs, VECs, LECs, T cells, and Mono/ Macro. However, three additional inflammatory cell clusters not found in non-DM skin were present in the skin of patients with DM: mast cells (marker tryptase $\alpha / \beta 1$ [TPSAB1]), natural killer (NK) cells (marker NK-cell granule protein 7 [NKG7]), and B cells (marker CD79A). This finding is consistent with the chronic inflammatory state of DM skin.

The cells from ulcers numbered 2,322 and were grouped into 11 clusters, which showed different composition compared with the previous two conditions (Fig. 4C and F). All markers are shown in Supplementary Table 12. Specifically, there were three instead of five fibroblast clusters: Fibro1 was characterized by high expression of panfibroblast markers proteoglycans $D C N, L U M$, and $V C N$ as well as reticular marker MFAP5 (11); Fibro2 was uniquely enriched for inflammatory genes IL11, IL24, MMP1, and MMP3 and similar to the DM condition for papillary markers COL7A1 and WNT5A; and Fibro3 exhibited high levels of MMP12 and, interestingly, macrophage markers CD68 and CHI3L1. A number of adipogenesis-related genes prominent in this cluster, such as TIMP1 (23) and PLIN2 (24), suggest that these cells were adipocyte associated. In 
addition, there were two SMC and two VEC clusters. Because these cells originate from an active wound, which explains the lower number of captured cells, the additional clusters could correspond to activated states of these cell types. Notably, the anatomical site of ulcers is on the plantar surface as opposed to non-DM and DM specimens that were mostly obtained from the dorsum of the foot, which could further contribute to observed differences.

We compared the proportions of cell types across conditions (Fig. 4H). The non-DM samples had a markedly high proportion $(>50 \%)$ of fibroblasts compared with the other groups. The DM samples had a higher proportion of $\mathrm{T}$ cells and additional inflammatory clusters, namely NK cells, B cells, and mast cells. Moreover, the DFU samples had a higher percentage of endothelial cells and SMCs.

To further investigate the heterogeneity of the most abundant cell type, fibroblast clusters were analyzed with Metascape (Supplementary Fig. 6). The non-DM clusters showed top enrichment of gene ontology (GO) terms collagen fibril organization, collagen formation, and growth factor binding, while the DM clusters exhibited response to wounding as the top-enriched GO term (Supplementary Fig. $6 C$ and $D$ ). This finding points to DM fibroblasts being "primed" with a wounding phenotype even before development of an ulcer. The top-enriched GO terms for DFU clusters were blood vessel development and ECM organization, indicating that their primary role in wounding is neoangiogenesis promotion. Of interest, a particular papillary fibroblast cluster that was only present in patients with DM and DFU was enriched for COL7A1. Type VII collagen is a major constituent of the basement membrane, a central structure of the dermal-epidermal junction, and plays a critical role in wound healing (25).

Next, we performed canonical correlation analysis (CCA) by combining all captured cells, which enabled comparisons between conditions. The resulting analysis yielded 14 clusters, which we identified as previously mentioned (Supplementary Fig. 7). All markers from the CCA are listed in Supplementary Table 13. Most of the clusters contained cells from all three conditions, with the exception of B cells and Fibro4, which were predominantly composed of DM and DFU cells (Supplementary Fig. 7D). Confirming our previous observations, cluster Fibro4 was enriched for COL7A1 and additional papillary markers COL6A1 and $P D P N$, suggesting the presence of a DM-specific fibroblast subpopulation.

\section{Exploring the Myeloid Cell Cluster}

Because monocytes and macrophages are integral players of the inflammatory response, we assessed the expression of established markers for monocytes (CD14, ITGAM, CSF1R) and macrophages (CD68, CXCR4) and found that the majority of the myeloid cell cluster cells were macrophages (Supplementary Fig. 8A), with a slight enrichment for monocytes in DFU. We then used a panel of markers for M1- or M2-associated macrophages to further characterize the polarization state of the cells. Initially, we used a cell scoring approach, where lists of published M1 or M2 marker genes $(26,27)$ were used to assign scores to each individual cell depending on its expression. Hence, a positive score would suggest that this module of genes is expressed in a particular cell more highly than expected given the average expression across the population. No significant fluctuations in the scores of different conditions were evident, indicating the same degree of general polarization (Supplementary Fig. 8B). The markers used are listed in Supplementary Table 14. To dissect the macrophages' polarized states, we looked at the expression of specific markers individually. For M1 markers, some were more highly expressed in DFU (CD86, IL6), while others were enriched in non-DM (TNF, IL1B). The DM samples had an expression pattern more similar to the non-DM rather than the DFU samples (Supplementary Fig. $8 C$ ). With regard to M2 markers, some were more prominent in DFU samples (CD163, SLAMF1), while others were upregulated in non-DM (MRC1, IL10) or DM (CD36, CCL18) samples (Supplementary Fig. 8D). These findings indicate that even though the general polarization appears similar, differences arise in the gene expression of particular markers.

\section{Differential Expression Analysis and IPA for Selected Cell Types}

For all cells that clustered in the same group following CCA processing, differential expression (DE) analysis was performed using model-based analysis of single-cell transcriptomics (28). DE analysis was conducted for all possible combinations (i.e., DFU vs. non-DM, DFU vs. DM, DM vs. non-DM). We then used IPA to predict upstream regulators that lead to the expression patterns found in the data set.

To select which clusters to prioritize for IPA, we used the markers identified to be associated with poor or enhanced healing by our serum measurements' PCA and examined their expression. Looking at the poor healingassociated markers, CXCL8 was more highly expressed in the DM conditions (purple and brown dots) and predominantly by Mono/Macro cluster. CCL3, CCL4, and TNF were likewise mainly expressed by Mono/Macro cluster (Fig. $5 A$ ). For enhanced healing markers, ICAM1 was more expressed by VECs, while MMP2 was present in all different fibroblast clusters but less so for DFU (smaller brown dots) (Fig. 5B). On the basis of these findings, we selected Mono/Macro and VEC clusters for further analysis.

In the myeloid cell cluster, DFU versus non-DM upstream regulator analysis showed a number of M1 markers activated in DFU, such as NOS2 (iNOS), IL-6, and TIMP1 (Fig. 5D red underlined), whereas M2 markers were downregulated, including MMP9 and IL-10 (green underlined). Furthermore, inhibited regulator analysis yielded IL-13 as a central inhibited master regulator (Fig. $5 E$ ). In DFU versus DM DE analysis, IL-13 was also inhibited (Fig. 5F). When comparing DM versus non-DM, 16 upstream regulators were predicted to be inhibited, leading in turn to 


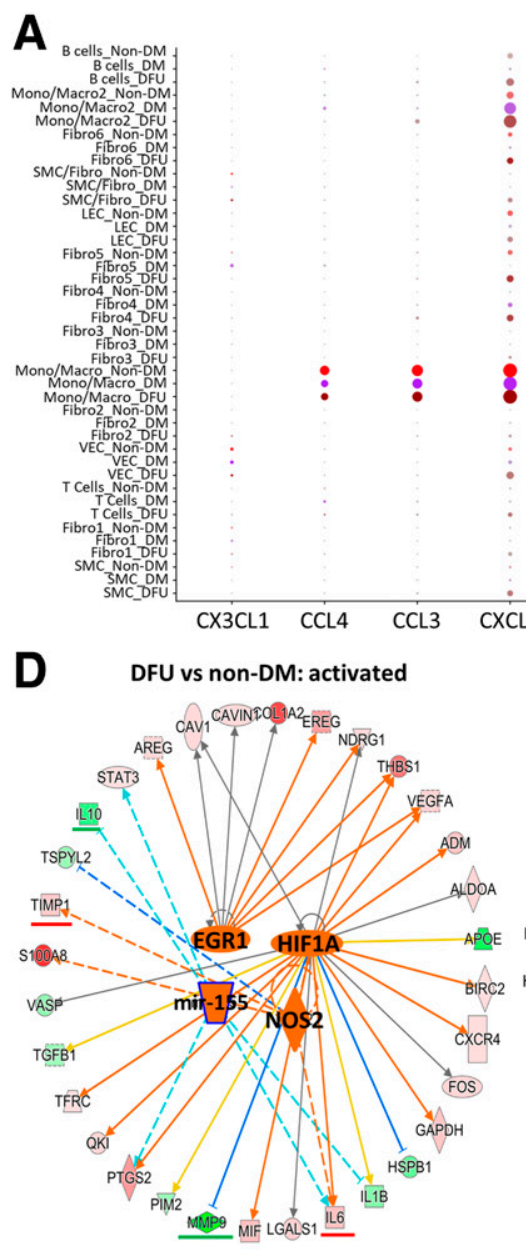

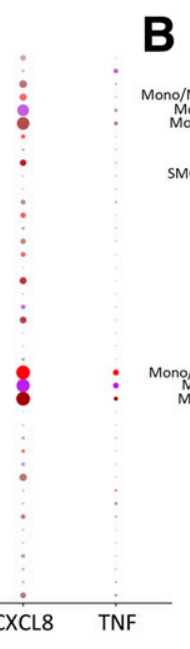

B

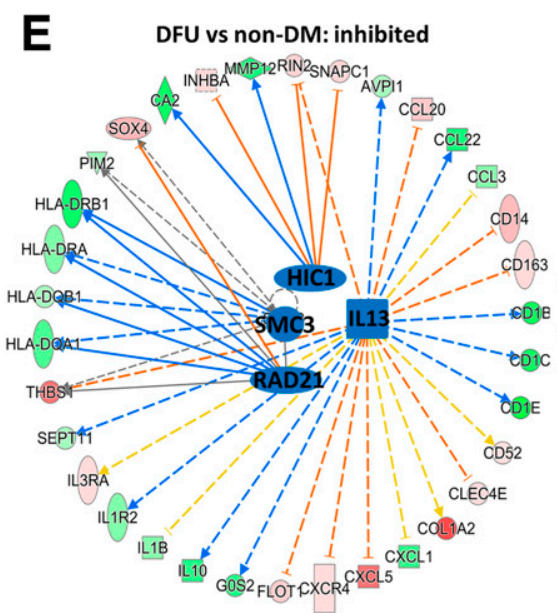

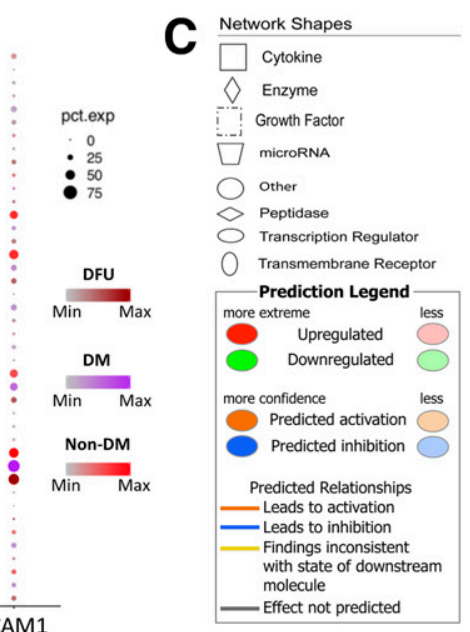

$\mathbf{F}$

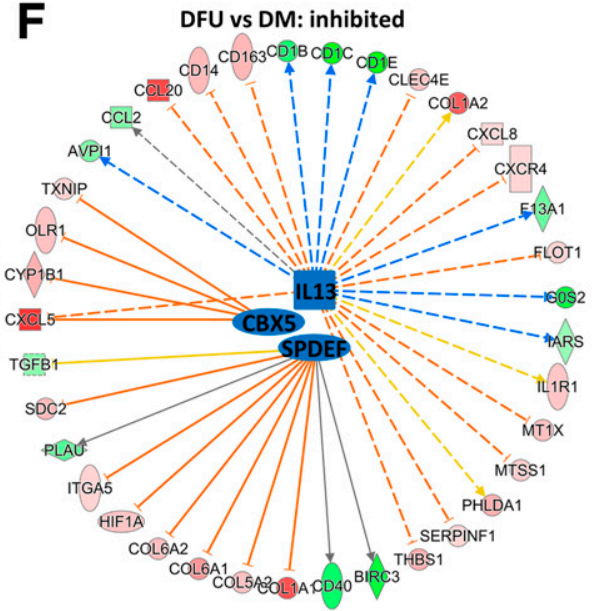

Figure 5-Serum cytokine and chemokine markers at single-cell level. $A$ : Serum markers associated with poor healing (CX3CL1, CCL4, CCL3, CXCL8, and TNF, from left to right on $x$-axis) and their expression in single-cell RNA-seq data on a split dot plot. $B$ : Serum markers associated with enhanced healing (IL-13, IL-10, MMP2, TAC1, VCAM1, and ICAM1, from left to right on $x$-axis) and their expression in singlecell RNA-seq data on a split dot plot. As outlined in the legend, the size of the dot encodes the percentage of cells within a class expressing the gene (pct.exp), different colors represent the conditions, and color intensity in the scale correlates with higher expression levels across all cells within a class. The $y$-axes in panels $A$ and $B$ list all the clusters computed from CCA per condition. $C$ : Detailed legend explaining IPA results. D: DFU vs. non-DM DE IPA for activated upstream regulators in myeloid cell cluster with EGR1, HIF1A, mir-155, and NOS2 as central regulators. E: DFU vs. non-DM DE IPA for inhibited upstream regulators in myeloid cells with HIC1, IL13, RAD21, and SMC3 as central regulators. F: DFU vs. DM DE IPA of inhibited upstream regulators in myeloid cells with CBX5, IL-13, and SPDEF as central regulators.

inhibition of biological processes, such as cell movement of monocytes, migration of dendritic cells, and chemotaxis of antigen-presenting cells (Supplementary Fig. 9). These results point to a dysregulated migratory profile of immune cells in DM skin.

For VEC, DM versus non-DM DE regulator analysis revealed inhibition of interferons (IFNG, IFNA2, and IFNL1)

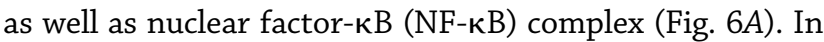
addition, DFU versus non-DM analysis pointed to inhibition of interferons in DFU (Fig. 6B). Similar results were obtained with DFU versus DM (Fig. 6C). This suggests impairment in IFNG and relevant genes in diabetes. We validated these findings at the protein level, showing reduced expression of IL-13 and IFN- $\gamma$ in DFU (Supplementary Fig. 10).

\section{Gene Signature of Healers and Nonhealers at the Single-Cell Level}

We next examined the gene expression distribution of the differentially expressed genes identified from bulk RNAseq analysis of nonhealers versus healers at the single-cell level. We found that SLCO2A1 and CYP1A1, which were upregulated in nonhealers, were mainly expressed by the VEC cluster almost exclusively in DFU, indicating a potential role in wound healing (Supplementary Fig. 11). For the list of genes upregulated in healers, high enrichment scores were notable in clusters Fibro2 and Fibro3 (Fig. 7), which mainly contained non-DM and DM cells. Representative genes coagulation factor XIII A chain (F13A1) and TNXB displayed elevated expression levels in Fibro3 and Fibro2, respectively (Fig. $7 B$ and $C$ ). Fibro2 is a reticular and Fibro3 
A
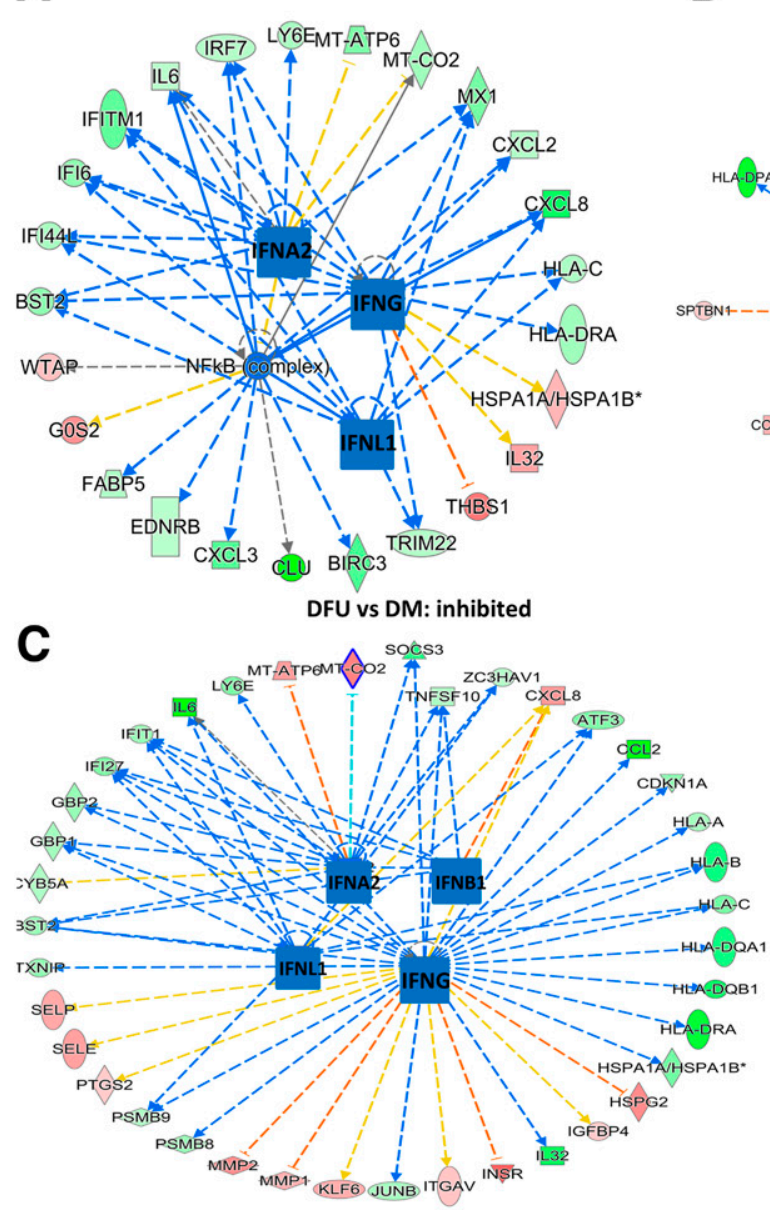

B

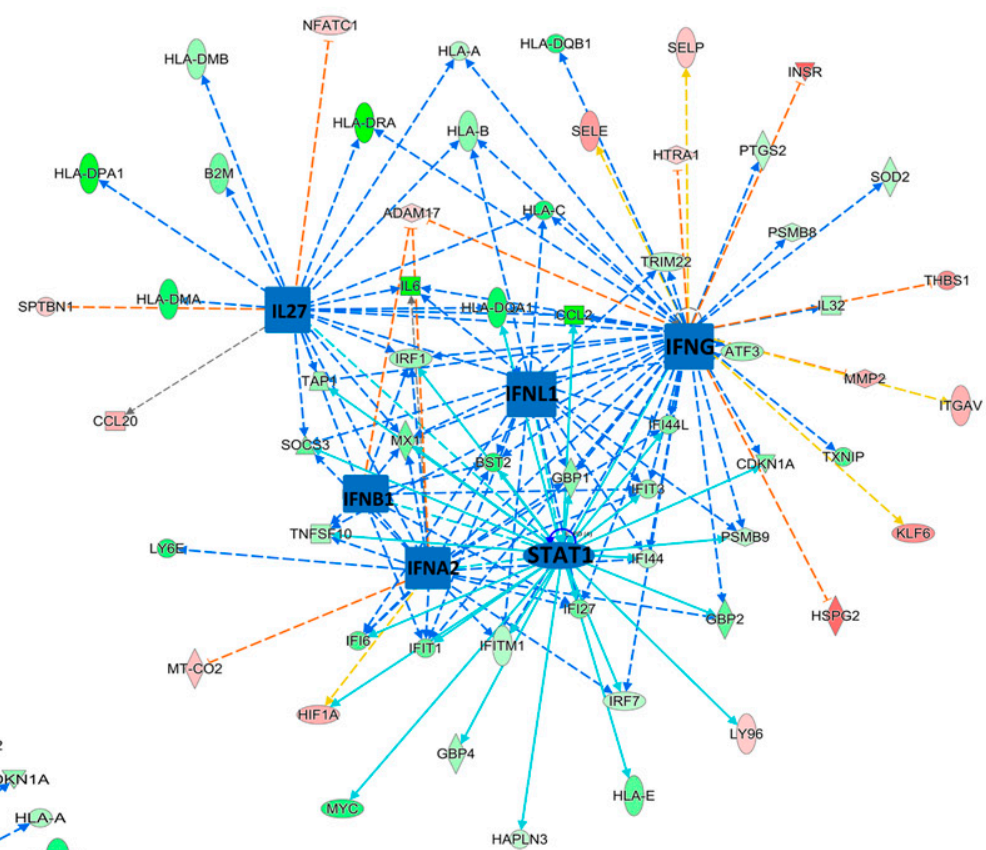

D

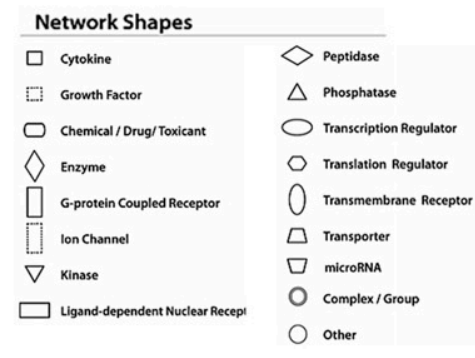

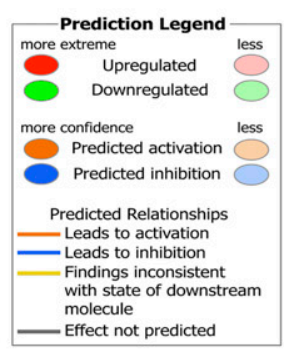

Figure 6-A: IPA of the VEC cluster. DM vs. non-DM DE IPA results for inhibited upstream regulators in VEC with IFNA2, IFNG, IFNL1, and NF- $\mathrm{B}$ (complex) as central regulators. B: DFU vs non-DM DE IPA analysis results for inhibited upstream regulators in VEC cluster with IFNA1, IFNB, IFNG, IFNL1, IL27, and STAT1 as central regulators. C: DFU vs. DM DE IPA results for inhibited upstream regulators in VEC cluster with IFNA2, IFNB1, IFNG, and IFNL1 as central regulators. D: Detailed legend explaining IPA results.

a papillary fibroblast cluster, suggesting that distinct fibroblast subpopulations are implicated in the pathogenesis of diabetes. Notably, both F13A1 and TNXB have been shown to be associated with wound healing $(29,30)$ and could represent both markers for prediction of ulcer healing and therapeutic intervention targets.

\section{DISCUSSION}

The purpose of our investigation was to use state-of-theart techniques for identifying systemic and local factors associated with DFU healing. Inflammatory biomarkers in serum have been shown to correlate with acute wound healing $(31,32)$. To this end, multiplex serological assays indicated sVCAM, VEGF, and IFN- $\gamma$ as important components correlating with healing. VEGF and sVCAM are angiogenesis regulators (33), suggesting that healing is characterized by more active vasculature formation. Even though IFN- $\gamma$ has been reported to inhibit wound healing in vivo $(34,35)$, rapid healing venous ulcers exhibited increased levels of IFN- $\boldsymbol{\gamma}$ (36). Impaired healing in DM is typically associated with chronic inflammation; nevertheless, we observed that a greater abundance of proinflammatory marker IFN- $\gamma$ correlates with DFU healing. This finding is in line with publications by us (37) and others (38), where conversion of chronic inflammation to acute is essential for successful healing. Further studies are necessary to fully understand the underlying mechanisms.

Both patients with type $1 \mathrm{DM}$ and patients with type 2 DM were included in the study. This was based on our previous reports that have shown that DFU pathophysiology does not differ between these two populations. We also did not observe any between-group difference in the concentration of the main serum cytokines and biomarkers of endothelial function.

Moreover, histologic assessment of forearm biopsies revealed that in agreement with our previous work, patients with DM exhibited an increased percentage of degranulated mast cells (4). In addition, the healers had more inflammatory cells and more alternatively activated 

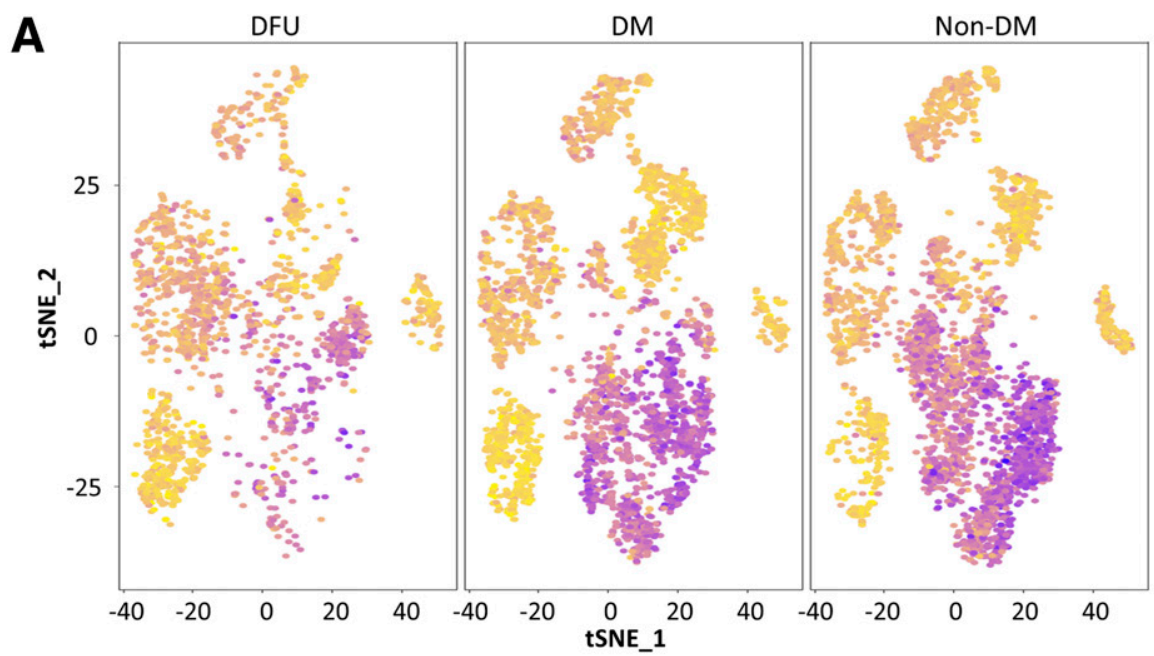

Enrichment Score Scaled Expression
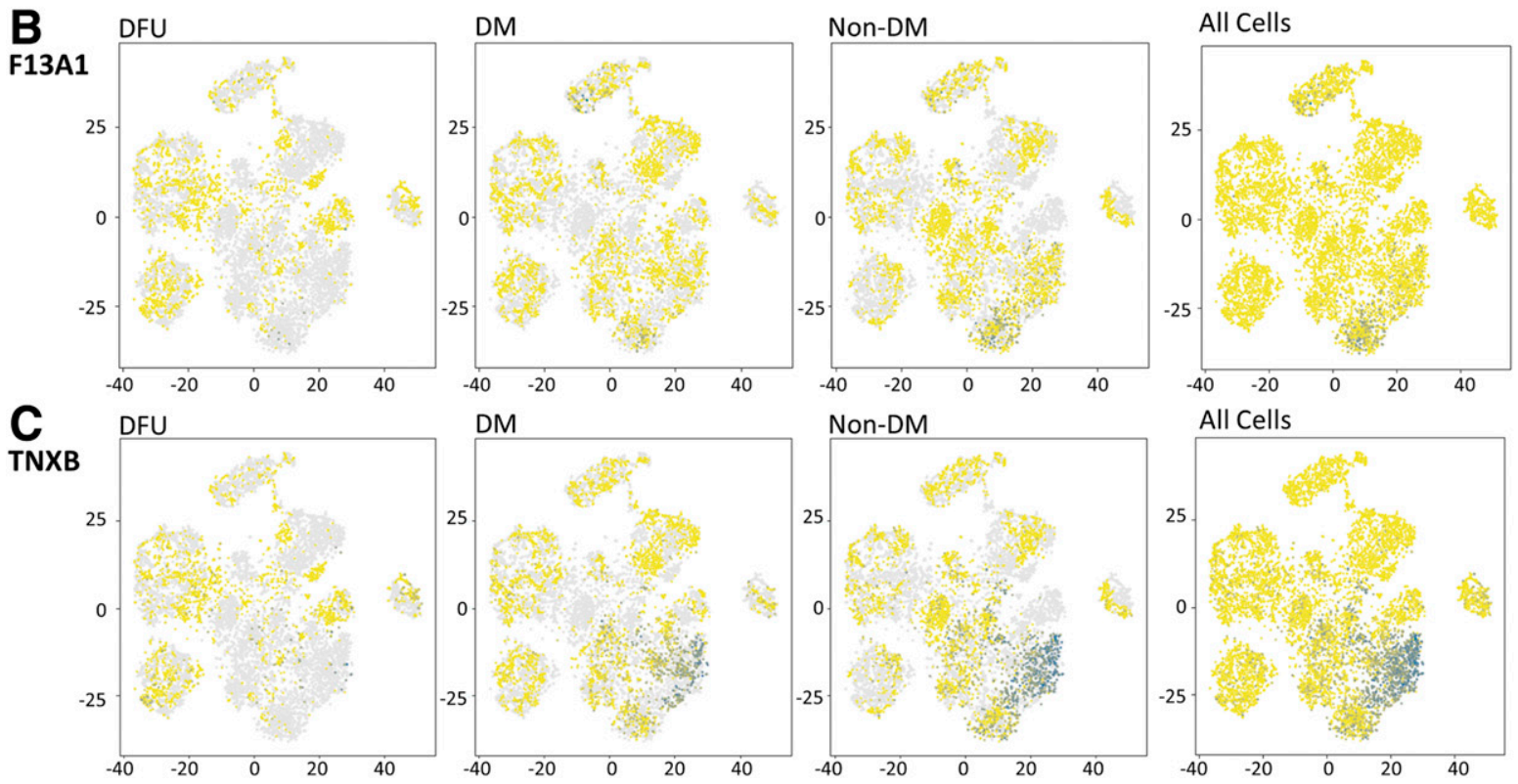

Figure 7-Differentially expressed genes (DEGs) upregulated in forearm skin healers' bulk RNA-seq samples at single-cell level. $A$ : Feature heat maps depicting the enrichment score generated from the list of all DEGs upregulated in forearm skin of patients who healed their ulcers. $t$-SNE plots with cells from each condition (DFU, DM, and non-DM). Each dot represents one cell, with purple denoting high enrichment score and intensity of color corresponding to increased scores, according to the scale shown. $B$ and $C$ : Representative selected DEGs expressed in the single-cell RNA-seq data. $t$-SNE plots of F13A1 expression $(B)$ and TNXB $(C)$ across the different conditions (DFU, DM, non-DM). All the cells are also shown together in the same plot. Each dot represents one cell, with yellow denoting no expression and green denoting high expression and intensity of color corresponding to increased expression levels, according to the scale shown. Gray dots correspond to nonpresent cells for the particular condition.

macrophages, demonstrating that DM affects skin systemically and not only at the foot (39).

To this direction, we hypothesized that exploring gene expression of forearm biopsies could offer insights into how systemic changes in DM affect skin at a nonulcerative site. Bulk RNA-seq analysis in nonhealers showed upregulation of CYP1A, which is expressed at sebaceous glands and is associated with skin barrier function (40), and SLCO2A1, which is highly abundant in VECs (41) and its inhibition leads to accelerated DM wound healing $(42,43)$. In healers, upregulated genes were mostly ECM related and included MMP2. MMP2 is elevated in chronic wounds (44), and higher expression by fibroblasts has been linked to more efficient ECM remodeling (45). Furthermore, IL13 and IFNG were the top implicated upstream regulators.

Finally, we conducted single-cell RNA-seq experiments where we surveyed the transcriptomes of a large number of cells from the foot skin of healthy subjects and patients with DM with and without DFU. Although studies have evaluated singe-cell transcriptomics of healthy (46) or diseased $(47,48)$ human skin, to our knowledge, this is the first report of single-cell RNA-seq on lower-extremity skin from such a population. In accordance with other studies 
$(11,49)$, we found several different fibroblast clusters, including a COL7A1-expressing population specifically enriched in DM skin. Chronic inflammation and hyperglycemia are hallmarks of DM, and Metascape analysis of DM fibroblasts suggested that continual exposure to these stressors confers an injury response-associated gene expression profile to the cells. DM skin also contained more inflammatory cells, which agrees with our current and previous histological observations.

In DFU samples, the higher proportion of both SMCs and VECs and the GO enrichment analysis of fibroblasts underscored that angiogenesis is a prominent process, although this does not mean that it is sufficient and can lead to complete healing. Additional research is required to explore the underlying mechanisms of dysfunction in DFU. In VEC and myeloid cell clusters, IL13 and IFN family members were found to be inhibited upstream regulators. This was corroborated with staining of foot skin samples. Altogether, our results from integrated protein and transcriptome analyses suggest that activation of IL13 and IFNG could potentially be harnessed for enhancing DFU healing.

The limitations of the study include not capturing any epidermal cells for single-cell RNA-seq and analyzing the ulcers as a whole. Future studies should focus on dissecting the contribution of cells from different wound compartments, including ulcer edges, center, and healthy adjacent skin. Additionally, because protein levels do not always correlate with gene expression, it would be useful to combine single-cell proteomics with RNA-seq (50) for a more comprehensive characterization of cellular heterogeneity in DFU.

Funding. This work was supported by National Institutes of Health (NIH) grant 1R01-DK-091949 (to A.V.) and National Insitute of Diabetes and Digestive and Kidney Diseases Diabetic Complications Consortium grant 17 AU3773 (to A.V.). A.V. received funding from the National Rongxiang Xu Foundation. G.T. received a George and Marie Vergottis Foundation Postdoctoral Fellowship. M.R. received funding from the Fondation AGIR pour les Maladies Chroniques. S.D. received funding from the German Research Foundation (DFG). The data analysis performed by R.S.K. was supported by Harvard Catalyst, the Harvard Clinical and Translational Science Center (NIH award UL1-RR-025758 and financial contributions from participating institutions).

The content is solely the responsibility of the authors and does not necessarily represent the official views of the National Center for Research Resources or NIH. Duality of Interest. No potential conflicts of interest relevant to this article were reported.

Author Contributions. G.T., D.B., M.R., A.T., S.D., B.S., W.Z., J.F., S.B., A.K., and M.B. obtained the data. G.T., M.R., R.S.K., D.H., S.H.S., N.A.P., and A.V. performed the data analysis. G.T., M.R., and A.V. wrote the manuscript. A.V. was responsible for the study concept and design. All authors participated in data interpretation and critical review of the manuscript and approved the final report. A.V. is the guarantor of this work and, as such, had full access to all the data in the study and takes responsibility for the integrity of the data and the accuracy of the data analysis.

Prior Presentation. Parts of this study were presented at the 77 th Scientific Sessions of the American Diabetes Association, San Diego, CA, 9-13 June 2017; the 78th Scientific Sessions of the American Diabetes Association, Orlando, FL, 22-26 June 2018; and the Wound Healing Society 2018 Annual Meeting, Charlotte, NC, 25-29 April 2018.

\section{References}

1. Gregg EW, Li Y, Wang J, et al. Changes in diabetes-related complications in the United States, 1990-2010. N Engl J Med 2014;370:1514-1523

2. Falanga V. Wound healing and its impairment in the diabetic foot. Lancet 2005;366:1736-1743

3. Dinh T, Tecilazich F, Kafanas A, et al. Mechanisms involved in the development and healing of diabetic foot ulceration. Diabetes 2012;61:2937-2947

4. Tellechea A, Leal EC, Kafanas A, et al. Mast cells regulate wound healing in diabetes. Diabetes 2016;65:2006-2019

5. Eming SA, Martin P, Tomic-Canic M. Wound repair and regeneration: mechanisms, signaling, and translation. Sci Transl Med 2014;6:265sr6

6. Conesa A, Madrigal P, Tarazona S, et al. A survey of best practices for RNAseq data analysis [published correction appears in Genome Biol 2016;17:181]. Genome Biol 2016;17:13

7. Butler A, Hoffman P, Smibert P, Papalexi E, Satija R. Integrating single-cell transcriptomic data across different conditions, technologies, and species. Nat Biotechnol 2018;36:411-420

8. Krämer A, Green J, Pollard J Jr, Tugendreich S. Causal analysis approaches in Ingenuity Pathway Analysis. Bioinformatics 2014;30:523-530

9. Zechner R, Moser R, Newman TC, Fried SK, Breslow JL. Apolipoprotein E gene expression in mouse 3T3-L1 adipocytes and human adipose tissue and its regulation by differentiation and lipid content. J Biol Chem 1991;266:10583-10588 10. White RT, Damm D, Hancock N, et al. Human adipsin is identical to complement factor $\mathrm{D}$ and is expressed at high levels in adipose tissue. J Biol Chem 1992;267:9210-9213

11. Philippeos C, Telerman SB, Oulès B, et al. Spatial and single-cell transcriptional profiling identifies functionally distinct human dermal fibroblast subpopulations. J Invest Dermatol 2018;138:811-825

12. Nauroy $P$, Barruche $V$, Marchand $L$, et al. Human dermal fibroblast subpopulations display distinct gene signatures related to cell behaviors and matrisome. J Invest Dermatol 2017;137:1787-1789

13. Haydont V, Neiveyans V, Fortunel NO, Asselineau D. Transcriptome profiling of human papillary and reticular fibroblasts from adult interfollicular dermis pinpoints the 'tissue skeleton' gene network as a component of skin chronoageing. Mech Ageing Dev 2019;179:60-77

14. Theocharidis G, Drymoussi Z, Kao AP, et al. Type VI collagen regulates dermal matrix assembly and fibroblast motility. J Invest Dermatol 2016;136:74-83

15. Kim HS, Lee J, Lee DY, et al. Schwann cell precursors from human pluripotent stem cells as a potential therapeutic target for myelin repair. Stem Cell Reports 2017;8:1714-1726

16. Paquet-Fifield $\mathrm{S}$, Schlüter $\mathrm{H}$, Li A, et al. A role for pericytes as microenvironmental regulators of human skin tissue regeneration. J Clin Invest 2009;119: 2795-2806

17. Matsui TS, Ishikawa A, Deguchi S. Transgelin-1 (SM22 $\alpha$ ) interacts with actin stress fibers and podosomes in smooth muscle cells without using its actin binding site. Biochem Biophys Res Commun 2018;505:879-884

18. Bevilacqua MP, Pober JS, Mendrick DL, Cotran RS, Gimbrone MA Jr. Identification of an inducible endothelial-leukocyte adhesion molecule. Proc Natl Acad Sci U S A 1987;84:9238-9242

19. Banerji S, Ni J, Wang SX, et al. LYVE-1, a new homologue of the CD44 glycoprotein, is a lymph-specific receptor for hyaluronan. J Cell Biol 1999;144: 789-801

20. Chtanova T, Newton R, Liu SM, et al. Identification of T cell-restricted genes, and signatures for different $\mathrm{T}$ cell responses, using a comprehensive collection of microarray datasets. J Immunol 2005;175:7837-7847

21. Alasoo K, Martinez FO, Hale C, et al. Transcriptional profiling of macrophages derived from monocytes and iPS cells identifies a conserved response to LPS and novel alternative transcription. Sci Rep 2015;5:12524

22. Montelius $A$, Marmigère $F$, Baudet $C$, Aquino JB, Enerbäck $S$, Ernfors $P$. Emergence of the sensory nervous system as defined by Foxs1 expression. Differentiation 2007;75:404-417 
23. Meissburger B, Stachorski L, Röder E, Rudofsky G, Wolfrum C. Tissue inhibitor of matrix metalloproteinase 1 (TIMP1) controls adipogenesis in obesity in mice and in humans. Diabetologia 2011;54:1468-1479

24. Takahashi Y, Shinoda A, Kamada H, Shimizu M, Inoue J, Sato R. Perilipin2 plays a positive role in adipocytes during lipolysis by escaping proteasomal degradation. Sci Rep 2016;6:20975

25. Nyström A, Velati D, Mittapalli VR, Fritsch A, Kern JS, Bruckner-Tuderman L. Collagen VII plays a dual role in wound healing. J Clin Invest 2013;123:3498-3509

26. Martinez F0, Gordon S, Locati M, Mantovani A. Transcriptional profiling of the human monocyte-to-macrophage differentiation and polarization: new molecules and patterns of gene expression. J Immunol 2006;177:7303-7311

27. Tang-Huau TL, Gueguen $P$, Goudot $C$, et al. Human in vivo-generated monocyte-derived dendritic cells and macrophages cross-present antigens through a vacuolar pathway. Nat Commun 2018;9:2570

28. Finak G, McDavid A, Yajima M, et al. MAST: a flexible statistical framework for assessing transcriptional changes and characterizing heterogeneity in singlecell RNA sequencing data. Genome Biol 2015;16:278

29. Egging D, van Vlijmen-Willems I, van Tongeren T, Schalkwijk J, Peeters A. Wound healing in tenascin- $X$ deficient mice suggests that tenascin- $X$ is involved in matrix maturation rather than matrix deposition. Connect Tissue Res 2007;48:93-98 30. Inbal A, Dardik R. Role of coagulation factor XIII (FXIII) in angiogenesis and tissue repair. Pathophysiol Haemost Thromb 2006;35:162-165

31. Hawksworth JS, Stojadinovic A, Gage FA, et al. Inflammatory biomarkers in combat wound healing. Ann Surg 2009;250:1002-1007

32. Csősz É, Tóth N, Deák E, Csutak A, Tőzsér J. Wound-healing markers revealed by proximity extension assay in tears of patients following glaucoma surgery. Int J Mol Sci 2018;19:4096

33. Fukushi J, Ono M, Morikawa W, Iwamoto Y, Kuwano M. The activity of soluble VCAM-1 in angiogenesis stimulated by IL-4 and IL-13. J Immunol 2000;165: 2818-2823

34. Laato M, Heino J, Gerdin B, Kähäri VM, Niinikoski J. Interferon-gammainduced inhibition of wound healing in vivo and in vitro. Ann Chir Gynaecol 2001; 90(Suppl. 215):19-23

35. Miles RH, Paxton TP, Zacheis D, Dries DJ, Gamelli RL. Systemic administration of interferon-gamma impairs wound healing. J Surg Res 1994;56:288-294 36. Beidler SK, Douillet CD, Berndt DF, Keagy BA, Rich PB, Marston WA. Inflammatory cytokine levels in chronic venous insufficiency ulcer tissue before and after compression therapy. J Vasc Surg 2009;49:1013-1020
37. Tellechea A, Bai S, Dangwal S, et al. Topical application of a mast cell stabilizer improves impaired diabetic wound healing. J Invest Dermatol 2020;140: 901-911.e11

38. Stone RC, Stojadinovic 0 , Rosa AM, et al. A bioengineered living cell construct activates an acute wound healing response in venous leg ulcers. Sci Transl Med 2017;9:eaaf8611

39. Van Hattem S, Bootsma AH, Thio HB. Skin manifestations of diabetes. Cleve Clin J Med 2008;75:772, 774, 776-777 passim

40. Rowe JM, Welsh C, Pena RN, Wolf CR, Brown K, Whitelaw CB. Illuminating role of CYP1A1 in skin function. J Invest Dermatol 2008;128:1866-1868

41. Topper JN, Cai J, Stavrakis G, et al. Human prostaglandin transporter gene (hPGT) is regulated by fluid mechanical stimuli in cultured endothelial cells and expressed in vascular endothelium in vivo. Circulation 1998;98:2396-2403

42. Syeda MM, Jing X, Mirza RH, Yu H, Sellers RS, Chi Y. Prostaglandin transporter modulates wound healing in diabetes by regulating prostaglandininduced angiogenesis. Am J Pathol 2012;181:334-346

43. Choi EY, Lee H, Woo JS, et al. Effect of onion peel extract on endothelial function and endothelial progenitor cells in overweight and obese individuals. Nutrition 2015;31:1131-1135

44. Wall SJ, Bevan D, Thomas DW, Harding KG, Edwards DR, Murphy G. Differential expression of matrix metalloproteinases during impaired wound healing of the diabetes mouse. J Invest Dermatol 2002;119:91-98

45. Stephens P, Davies KJ, Occleston N, et al. Skin and oral fibroblasts exhibit phenotypic differences in extracellular matrix reorganization and matrix metalloproteinase activity. Br J Dermatol 2001;144:229-237

46. Finnegan A, Cho RJ, Luu A, et al. Single-cell transcriptomics reveals spatial and temporal turnover of keratinocyte differentiation regulators. Front Genet 2019; 10:775

47. Apostolidis SA, Stifano G, Tabib T, et al. Single cell RNA sequencing identifies HSPG2 and APLNR as markers of endothelial cell injury in systemic sclerosis skin. Front Immunol 2018;9:2191

48. Tirosh I, Izar B, Prakadan SM, et al. Dissecting the multicellular ecosystem of metastatic melanoma by single-cell RNA-seq. Science 2016;352:189-196

49. Tabib T, Morse C, Wang T, Chen W, Lafyatis R. SFRP2/DPP4 and FM01/LSP1 define major fibroblast populations in human skin. J Invest Dermatol 2018;138 802-810

50. Stoeckius M, Hafemeister C, Stephenson W, et al. Simultaneous epitope and transcriptome measurement in single cells. Nat Methods 2017;14:865-868 